\title{
ANÁLISE PARAMÉTRICA DE PROTÓTIPOS DE MOVIMENTOS DE MASSA NA EVOLUÇÃO DE VERTENTES
}

\author{
Antonio Carlos Colangelo ${ }^{(a)}$ \\ (a) Departamento de Geografia/Faculdade de Filosofia, Letras e Ciências Humanas, Universidade de São Paulo, E- \\ mail: accolang@usp.br
}

\section{EIXO: SISTEMAS GEOMORFOLÓGICOS: ESTRUTURA, DINÂMICAS E PROCESSOS}

\begin{abstract}
Resumo
A parametrização de variáveis que intervêm nos processos de movimentos de massa apresenta muitas dificuldades. Além do grande número de variáveis envolvidas, elas devem ser avaliadas abaixo da superfície do terreno, nas proximidades de uma superfície teórica denominada "superfície de ruptura potencial" (SRP). Na tentativa de explorar essa complexidade, foram criados o "Emulador de Unidades de Vertentes" e o "Simulador de Estabilidade de Vertentes", dispositivos que operam de modo integrado, a partir de um conjunto de modelos geomórficos, geotécnicos e hidrológicos a eles incorporados. Com esses dispositivos digitais é possível criar uma infinidade de cenários paramétricos para estudar a estabilidade de encostas em sistemas de vertente sintéticos, a partir de 16 variáveis de entrada. Quando o fator de segurança (Fs) assumir valores abaixo da unidade para uma porção da "superfície de ruptura potencial", o simulador gera um protótipo de movimento de massa caracterizado por 69 parâmetros de saída.
\end{abstract}

Palavras chave: geomorfossíntese; geomorfocinemática; evolução de vertentes; estabilidade de vertentes.

\section{O Emulador de Unidades de Vertentes "EUV"}

O emulador de unidades de vertentes "EUV" é uma ferramenta capaz de gerar unidades sintéticas de vertentes em três dimensões a partir de um único perfil inicial. A esse perfil inicial é atribuído o status de perfil-geratriz (Fig.01a). A partir dele são gerados, por sucessivas iterações, novos perfis, os perfis descendentes, que são novos perfis de equilíbrio calculados a partir dos modelos incorporados ao emulador de unidades de vertentes. Por sua vez, um conjunto de perfis-descendentes dá origem a uma unidade sintética de vertente, a vertente descendente (Fig.02). Ao perfil de vertente geratriz foram incorporados modelos morfológicos, hidrológicos e geotécnicos, de tal modo que é possível projetar sua evolução no tempo e no espaço a partir da manipulação de um conjunto de 16 variáveis principais. Estas, interferem direta ou indiretamente nas condições de estabilidade da vertente e, portanto, sobre seu limiar de declividade (Colangelo, 2007; 2009)

Tal como foi concebido, o perfil-geratriz aproxima-se do conceito de vertente-limiar estabelecido por Carson (1975). Entretanto diverge dele porque, enquanto Carson entende a vertente-limiar como no limite 
de equilíbrio em toda sua extensão, no caso dos perfis-geratrizes e descendentes, tal condição pode estar circunscrita a apenas uma porção de sua extensão, estando o restante aquém dos limiares calculados. O quadro 1 adiante apresenta os dez principais modelos que estão incorporados ao "emulador de unidades de vertente" (EUV), e ao "simulador de estabilidade de vertentes" (SEV). Além deles, foram também incorporados índices que controlam fatores geomorfológicos tais como: o rebaixamento do nível-de-base, o recuo da vertente e o recuo das cabeceiras de drenagem.

Quadro 1 - Os 10 principais modelos incorporados ao emulador de unidades de vertente (EUV) e ao simulador de estabilidade de vertentes (SEV).

Estresse efetivo (Terzaghi, 1925)

Resistência ao cisalhamento (Coulomb, 1776 - Terzaghi, 1938)

Resistência ao cisalhamento (Fredlund, 1978)

Nível de saturação (Colangelo, 2007)

Profundidade da SRP (Colangelo, 2007)

Profundidade crítica da SRP

Declividade crítica da superfície de ruptura potencia

Nível crítico de saturação do solo

Projeção da declividade do topo do fluxo de percolação sobre a SRP (Chowdhury, 1978)

Peso específico do solo úmido $m_{c}=\left[\left(\left(\left(c-\gamma_{v} \cdot h_{v} \cdot \cos 2 \alpha \cdot(\tan \alpha-\tan \phi)\right) / Z_{c}\right)-\left(\gamma_{s}+\gamma_{w} \cdot n\right) \cdot \cos 2 \alpha \cdot(\tan \alpha-\tan \phi)\right) /\left(\gamma_{w} \cdot m \cdot W \cdot \tan \phi\right)\right] \quad[8]$

$$
\begin{array}{r}
\sigma^{\prime}=\left(\sigma_{\mathrm{n}}-\mathrm{u}_{\mathrm{w}}\right) \\
\mathrm{s}=\mathrm{c}^{\prime}+\left(\sigma_{\mathrm{n}}-\mathrm{u}_{\mathrm{w}}\right) \cdot \tan \phi \\
\mathrm{s}=\mathrm{c}^{\prime}+\left(\sigma_{\mathrm{n}}-\mathrm{u}_{\mathrm{a}}\right) \tan \phi^{\prime}+\left(\mathrm{u}_{\mathrm{a}}-\mathrm{u}_{\mathrm{w}}\right) \tan \phi_{\mathrm{b}}
\end{array}
$$

$\left.Z_{P R S}=\left[\tan \left((83,4-\beta)^{*} \cos \beta^{\star} 50+((A+B) / 2-100)-B\right) / 2,2\right)+0,0166\right] \quad[5]$

$Z_{c}=\left[\left(c-\gamma_{v} \cdot h_{v} \cdot \cos 2 \alpha \cdot(\tan \alpha-\tan \phi)\right) /\left(\left(\gamma_{s}+\gamma_{w} \cdot n\right) \cdot \cos 2 \alpha \cdot(\tan \alpha-\tan \phi)+\gamma_{w} \cdot m \cdot W \cdot \tan \phi\right)\right] \quad[6]$

$\alpha_{c}=\left[\arctan \left(\left(c+\left(\sigma-\gamma_{w} \cdot m \cdot Z \cdot W \cdot \tan \phi\right)\right) / \sigma\right)\right] \quad[7]$

$\mathrm{W}=\cot \alpha /(\tan \theta-\cot \alpha)$

$\gamma_{s w}=\left[\rho_{w} \cdot m \cdot I_{v}+\rho_{w} \cdot n \cdot I_{v}+\rho_{s} \cdot\left(1-I_{v}\right)\right] \quad[10]$

O dispositivo está concebido da seguinte maneira: numa primeira etapa é sintetizada uma unidade de vertente em 3 dimensões espaciais e uma temporal, a partir do perfil-geratriz preliminarmente determinado. Qualquer alteração em qualquer das variáveis ou fatores referidos no parágrafo anterior, que modifique o estado de equilíbrio original do perfil-geratriz, produz um novo perfil de equilíbrio, ou perfildescendente, cujas declividades correspondem agora àquelas dos limiares recalculados. Assim, para uma dada variável, é estabelecida uma taxa de variação que, se aplicada em sucessivas iterações gera uma coleção de novos perfis, os quais compõem uma unidade de vertente em 3D: a vertente-descendente.

Numa segunda etapa, a partir da aplicação do mesmo conjunto de modelos são avaliadas as condições de estabilidade ao longo da superfície de ruptura potencial "SRP", agora vinculada à vertente-descendente resultante. Assim, quando e onde os limiares de estabilidade forem atingidos, ou seja, os fatores de segurança estiverem abaixo da unidade $\left(\mathrm{F}_{\mathrm{S}}<1\right)$, o dispositivo identifica e delimita uma superfície que chamamos de superfície de efetivo potencial de ruptura "SEPR", ao mesmo tempo que faz desencadear um evento protótipo de movimentos de massa. 

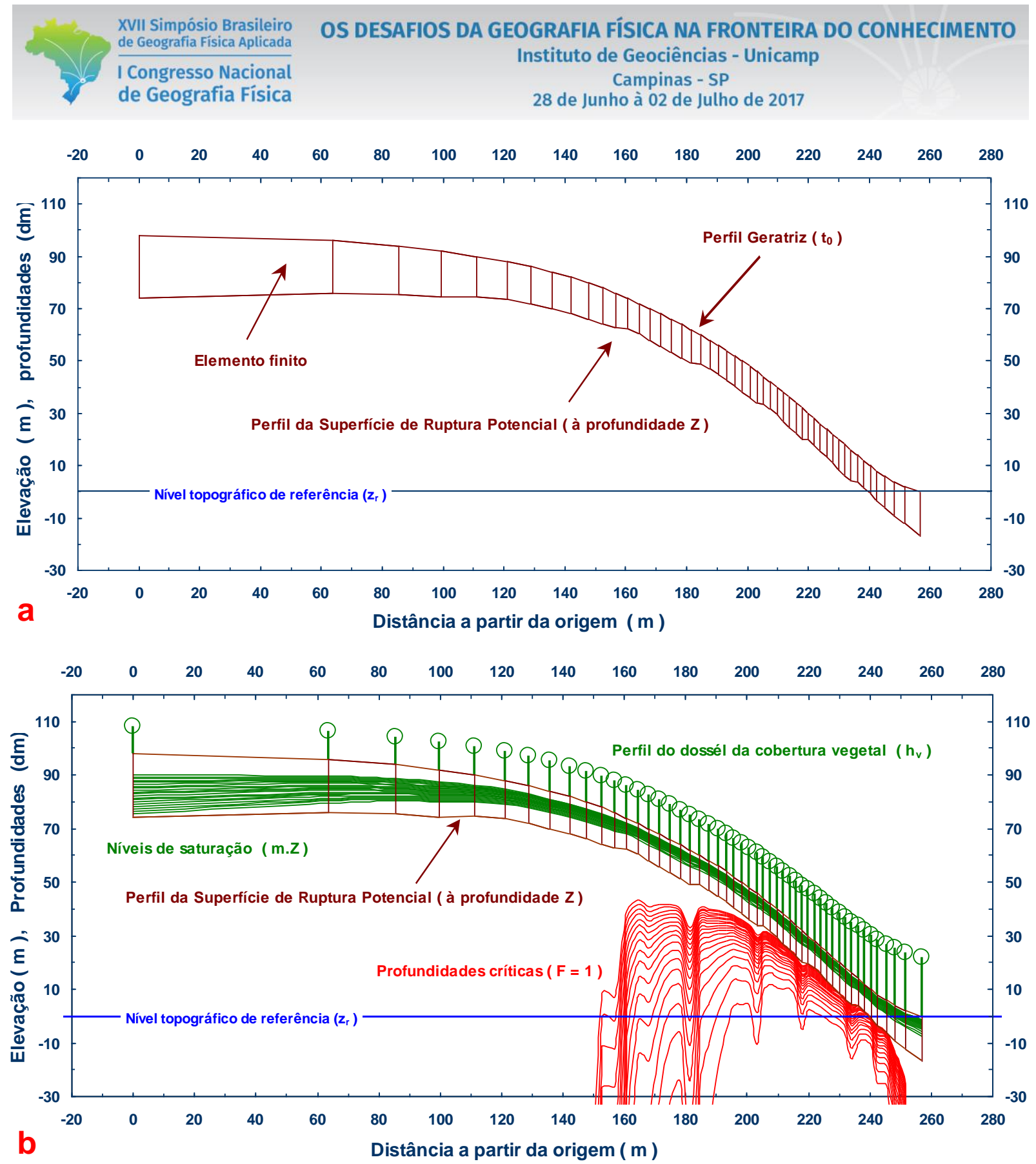

Fig.01 - Exemplo de perfil-geratriz. Em "a" exibindo o perfil da superfície de ruptura potencial e os elementos finitos, método a ser utilizado na análise do limite do equilíbrio. Em "b" o mesmo prefil com os níveis de saturação do solo, a estatura do dossél da cobertura vegetal, o equivalente em níveis da água retida nos bolsões texturais e as profundidades críticas calculadas pelo simulador de estabilidade.

O emulador de unidades de vertentes, EUV, trabalha de modo integrado ao simulador de estabilidade de vertentes, SEV, sendo possível gerar uma infinidade de quadros paramétricos e seus respectivos protótipos de movimentos de massa. Esses protótipos de eventos de movimentos de massa são descritos pelo simulador de estabilidade através de 69 parâmetros, o que permite uma análise quantitativa muito detalhada de suas características morfométricas, geotécnicas e hidrológicas (Tabelas 1 e 2).Não se trata de 


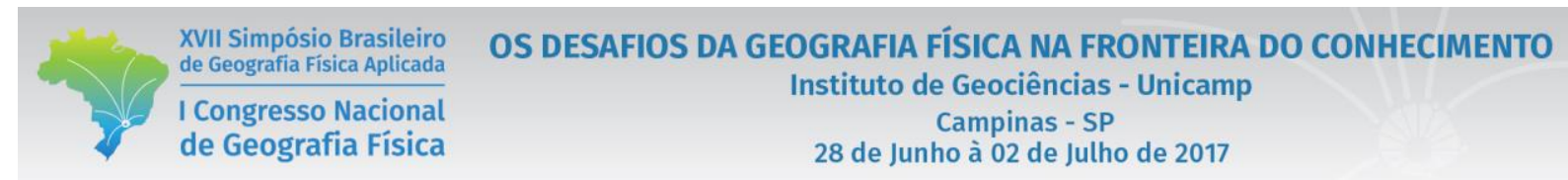

uma parametrização de eventos de movimentos de massa convencional, com vistas a um prognóstico elaborado a partir de condições empíricas específicas. Ao contrário, parte-se da montagem de quadros paramétricos teóricos obtidos de experimentos em ambiente digital, realizados a partir de um conjunto de modelos determinísticos e estatísticos (Quadro 01). Alguns referenciais empíricos foram também utilizados. Valores obtidos de medições e ensaios de campo e laboratório de variáveis físicas e geotécnicas vinculadas aos materiais terrígenos, tais como resistência ao cisalhamento (coesão e ângulo de fricção interna), densidade aparente do solo e as profundidades da cobertura pedológica e do regolito, serviram como referencias para os experimentos digitais realizados com o simulador.
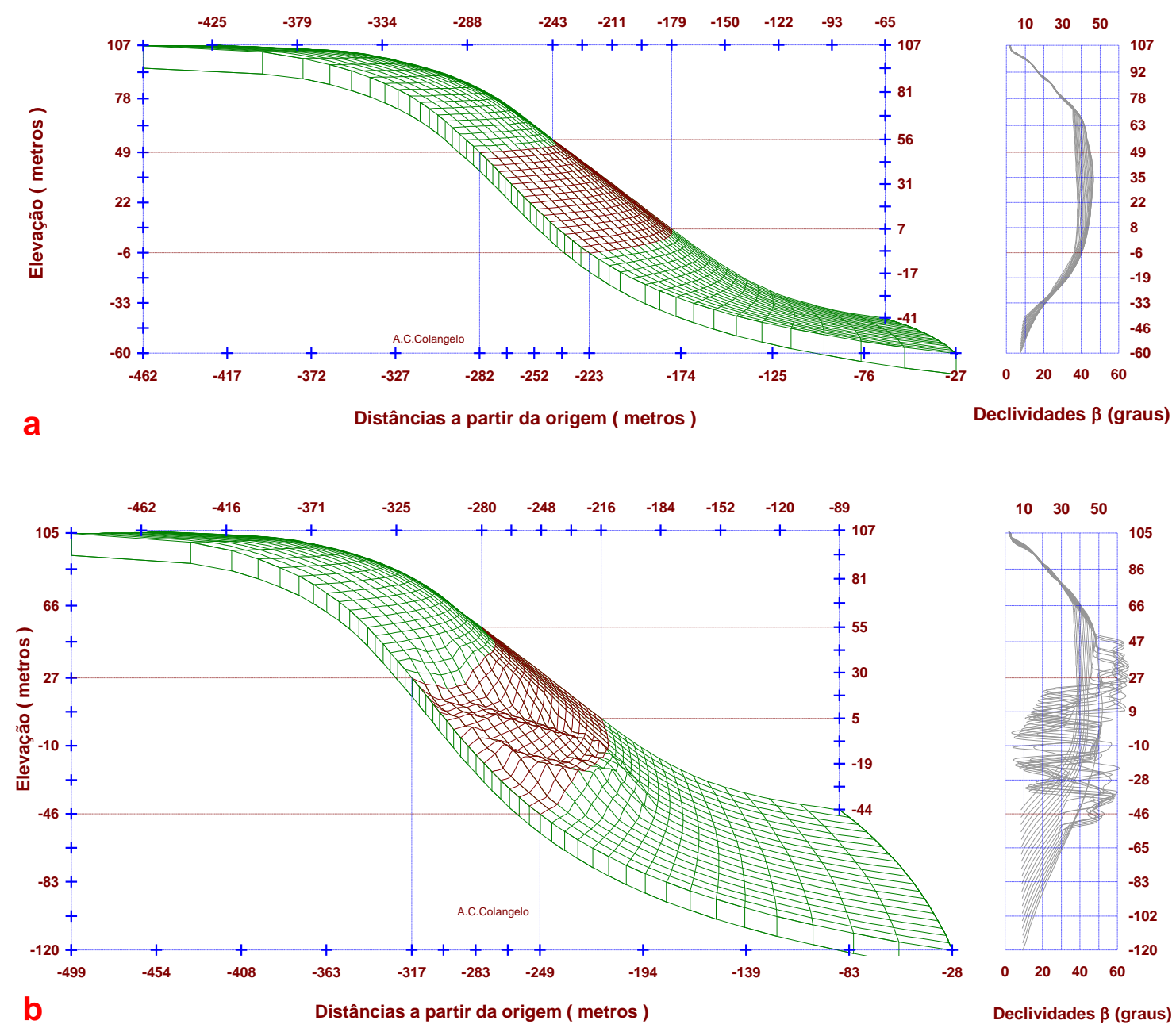

Fig.02 - Em "a", vertente sintética com amplitude topográfica interfluvial de $167 \mathrm{~m}$ e rebaixamento de nível-de-base da ordem de 19 m. Em "b", com 225 m de amplitude topográfica interfluvial, $86 \mathrm{~m}$ de rebaixamento de nível-de-base 


\section{OS DESAFIOS DA GEOGRAFIA FÍSICA NA FRONTEIRA DO CONHECIMENTO \\ Instituto de Geociências - Unicamp \\ Campinas - SP \\ 28 de Junho à 02 de Julho de 2017}

\section{A variação do peso específico do solo e as alterações na estabilidade da encosta}

O simulador de estabilidade de encostas foi concebido segundo o modelo de ruptura de Mohr-Coulomb. Supõe portanto que o material tenha características reológicas similares às dos materiais terrígenos. Quer dizer, seja granular, heterométrico e anisotrópico, o que implica porosidade, densidade aparente e estrutura variáveis no espaço e no tempo. Nesse caso, as condições de ruptura são definidas, ao mesmo tempo, como estando no limite superior do equilíbrio de deformação e no limite inferior dos estados de ruptura (Anderson; Richards, 1987).

A densidade aparente do solo está relacionada à estrutura, composição textural e também à mineralogia dos materiais. Suas variações, no tempo e no espaço, são consequência da atuação simultânea dos mecanismos de alteração da rocha, da pedogênese e da geomorfogênese, sempre com forte participação da atividade biológica, principalmente nos ambientes úmidos. Tais variações, atuando diretamente sobre a carga do material sobrejacente à superfície de ruptura potencial, intervêm diretamente sobre a tensão cisalhante e sobre a resistência ao cisalhamento, portanto, sobre as condições de estabilidade da vertente, podendo favorecê-la ou desfavorecê-la conforme os valores apresentados pelo ângulo de fricção interna do material " $\phi$ ” frente à declividade da superfície de ruptura potencial “ $\alpha$ ”(Quadro 2). Essa relação não é nada intuitiva, podendo ser deduzida da equação da profundidade crítica que consta do Quadro 1.

Quadro 2 - Relações entre gradiente de peso específico do solo $\Delta \gamma_{\mathrm{s}}$ e a estabilidade da vertente vinculada às variações da profundidade crítica $Z_{\mathcal{C}}$, para as condições de bônus ou déficit de fricção (vide Quadro 1, equação 06).

\begin{tabular}{|c|c|c|}
\hline \multirow{2}{*}{$\begin{array}{c}\text { Gradiente de } \\
\text { peso específico } \\
\text { do solo }\end{array}$} & \multicolumn{2}{|c|}{$\begin{array}{c}\text { Ângulo de Ficção Interna }(\phi) \text { versus } \\
\text { Declividade da "SRP" }(\alpha)\end{array}$} \\
\cline { 2 - 3 } & $\begin{array}{c}\text { Bônus de fricção } \\
(\phi>\alpha)\end{array}$ & $\begin{array}{c}\text { Déficit de fricção } \\
(\phi<\alpha)\end{array}$ \\
\hline$\Delta \gamma_{\mathrm{S}}(+)$ & $\begin{array}{c}\Delta \mathrm{Z}_{\mathrm{c}}(+) \\
\text { maior estabilidade }\end{array}$ & $\begin{array}{c}\Delta \mathrm{Z}_{\mathrm{c}}(-) \\
\text { menor estabilidade }\end{array}$ \\
\hline$\Delta \gamma_{\mathrm{S}}(-)$ & $\begin{array}{c}\Delta \mathrm{Z}_{\mathrm{c}}(-) \\
\text { menor estabilidade }\end{array}$ & $\begin{array}{c}\Delta \mathrm{Z}_{\mathrm{c}}(+) \\
\text { maior estabilidade }\end{array}$ \\
\hline
\end{tabular}

Nas sub-regiões da superfície de ruptura potencial que apresentam bônus de fricção $(\phi>\alpha)$, se houver queda na densidade do material, perda de carga portanto, haverá aumento da instabilidade nas porções de 
encosta que com elas guardam correspondência (Quadro 2). Esta relação, perda de carga com aumento de instabilidade da encosta, está longe de ser intuitiva. Entretanto é facilmente deduzida do modelo de ruptura descrito pela equação de Coulomb-Terzaghi quando aplicada à análise do limite de equilíbrio (Quadro 1, equação 02).

Isso decorre do fato da tensão normal efetiva ("effective stress”) estar relacionada pela função tangente, tanto com o ângulo de fricção interna $(\phi)$ quanto com o ângulo de mergulho da superfície de ruptura potencial $(\alpha)$. Quer dizer, qualquer perda de carga, como por exemplo por redução do peso específico do material sobrejacente, reduz a tensão normal efetiva $\left(\sigma^{\prime}\right)$ e, consequentemente o atrito mobilizado $\left(\sigma^{\prime} \cdot \tan \phi\right)$, numa proporção maior que a redução observada na tensão cisalhante $(\tau \cdot \tan \alpha)$.

A condição crítica, ou limiar, pressupõe fator de segurança unitário $(\mathrm{F}=1)$. Sendo assim, é possível avaliar as profundidades, ou espessuras, críticas $Z_{c}$ (Quadro 1, equação 06) suportadas pelo material terrígeno nas proximidades da superfície de ruptura potencial, a partir das quais o material está em condições de sofrer ruptura (Colangelo, 2008; 2009).

Os resultados do experimento 455, exibidos na figura 3, são descritos por meio de 69 parâmetros apresentados nas tabelas 1 e 2. Trata-se de parâmetros morfológicos, geotécnicos e hidrológicos, cuja evolução no tempo está vinculada à evolução dos materiais superficiais inconsolidados de vertentes, tais como alteritas, depósitos coluviais e coberturas pedológicas. Por sua vez, a evolução desses mesmos materiais está condicionada à evolução dos fatores climáticos no decorrer do período Quaternário. Considerando essa escala de tempo, mesmo se não houver queda apreciável dos valores da coesão do material nas proximidades de uma possível superfície de ruptura potencial projetada, pode haver instabilização nos locais onde os ângulos de fricção interna são maiores que as declividades da "SRP”, em consequência da progressiva perda de peso específico do material a ela sobrejacente, como mostra o esquema representado no Quadro 2. Tais perdas podem ser resultantes, seja da atuação dos mecanismos de alteração da rocha, como no caso da alteração isovolume, seja daqueles vinculados à pedogênese, como ocorre nos horizonte de solo submetidos ao mecanismo de eluviação (Quadro 2).

O simulador de estabilidade de vertente possibilita examinar a evolução das condições de estabilidade ao longo da "SRP", testando relações entre variáveis tais como as anteriormente referidas. Na figura 3 estão representados três estágios de evolução das condições de estabilidade na referida unidade de vertente gerada pelo EUV. As consequências geomórficas e sedimentológicas imediatas da perda de estabilidade sofrida pelo material são exibidas pelo movimento de massa rotacional representado nesta figura. 

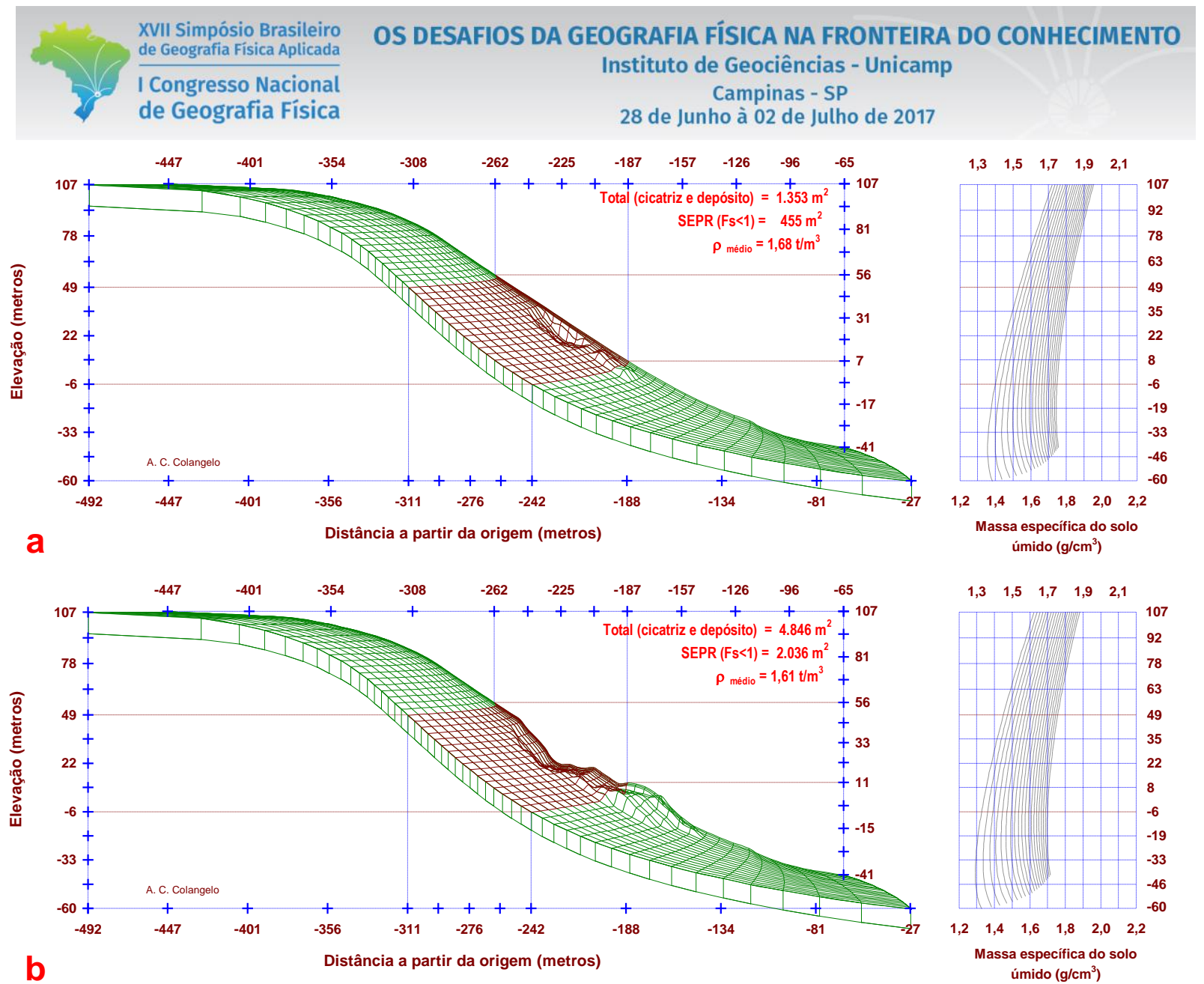

b

Distância a partir da origem (metros)
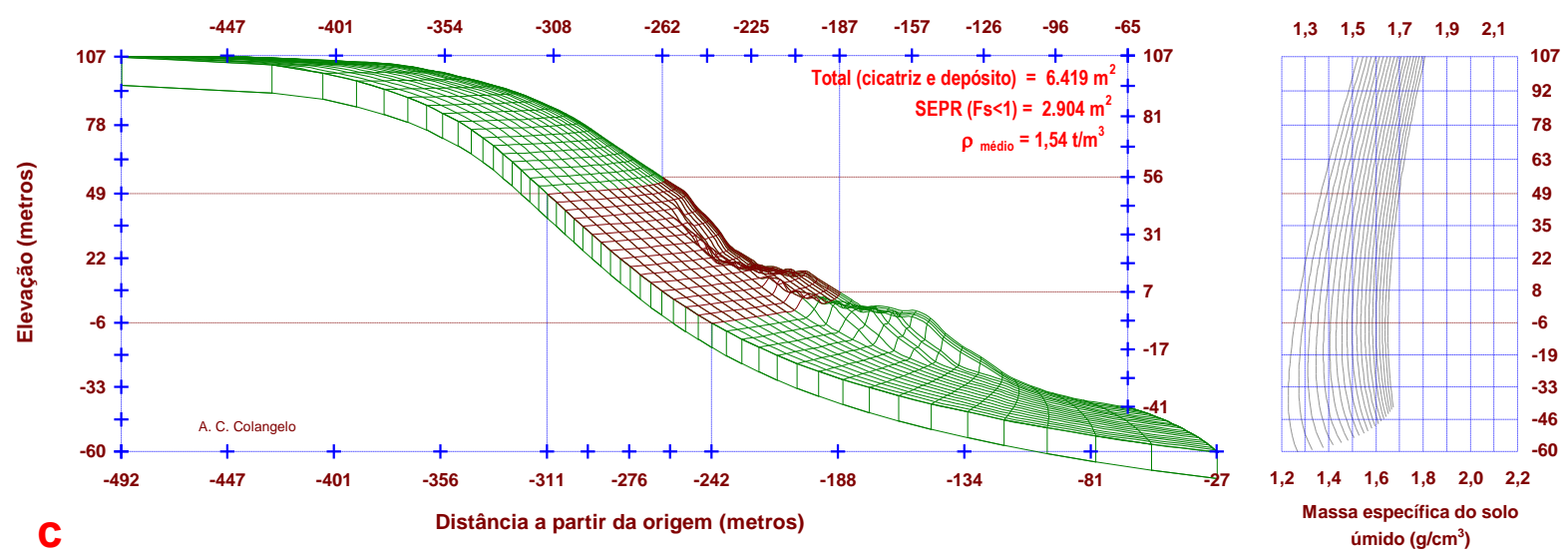

Figura 3 - Experimento 455. Três estágios de estabilidade "a", "b", "c", correpondentes à queda nos valores da densidade aparente do solo úmido, fixadas as outras variáveis. Em "a", ocorrência de pequeno deslizamento resultante de queda na densidade média do solo $\rho_{\mathrm{S}}$ de $1,72 \mathrm{t} / \mathrm{m}^{3}$ para $1,68 \mathrm{t} / \mathrm{m}^{3}(2,3 \%)$. Em "b", queda de $\rho_{\mathrm{S}}$ para $1,61 \mathrm{t} / \mathrm{m}^{3}\left(6,4 \%\right.$, em relação à condição inicial). Em "c", queda $\rho_{\mathrm{S}}$ médio para $1,54 \mathrm{t} / \mathrm{m}^{3}(10,5 \%)$. À direita, o comportamento da massa específica do solo úmido em relação ao posicionamento topográfico na vertente.

Devemos lembrar que cada um dos estágios, "a", "b", "c", representa uma situação geomorfocinemática independente, de modo que não há uma conexão dinâmica entre os estágios apresentados. Lembramos que 


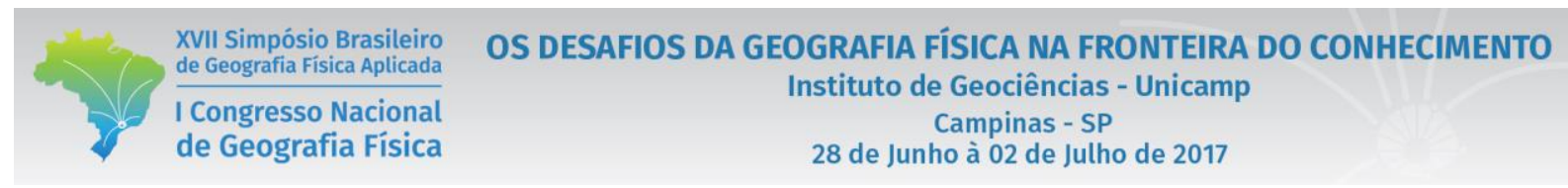

cada um dos infinitos possíveis estágios de evolução apresentados por um protótipo de movimento de massa obtido a partir desse dispositivo representa uma condição de equilíbrio vinculada a um quadro paramétrico específico. Desta forma, uma coleção destes estágios paramétricos gera uma sequência geomorfocinemática tridimensional. Sabemos que, para as condições efetivas de ocorrência de eventos de movimentos de massa, devem ser consideradas também as relações reológicas tensão-deformação (Hvorslev, 1936) e os gradientes de força e tensão vinculados à aceleração da massa de material terrígeno. Neste experimento, a área de ocorrência de deficit de fricção $(\phi<\alpha)$ não coincide com a área de deficit de resistência ao cisalhamento, como podemos observar. Portanto, ela também não coincide com a superfície de efetivo potencial de ruptura "SEPR" $(\mathrm{F}<1)$, a partir da qual é desencadeado o protótipo de movimento de massa (Figuras. 3c). Devemos salientar também que a "SEPR" não coincide com as porções de maior declividade da superfície de ruptura potencial "SRP”, nem tampouco com as porções de maior declividade da superfície do terreno (Colangelo, 2007; 2012).

Tabela 1 - Parâmetros físicos, morfométricos e topográficos referentes à unidade sintética de vertente e ao protótipo de movimento de massa gerados pelo experimento 455 .

\begin{tabular}{|c|c|c|c|c|}
\hline \multirow{8}{*}{$\begin{array}{l}\text { UNIDADE SINTÉTICA DE } \\
\text { VERTENTE }\end{array}$} & \multirow{4}{*}{ MORFOMETRIA } & Declividade média $\left(\beta_{\mathrm{m}}\right)$ & \multicolumn{2}{|l|}{27,58 graus } \\
\hline & & Declividade máxima $\left(\beta_{\max }\right)$ & \multicolumn{2}{|l|}{42,92 graus } \\
\hline & & Comprimento médio (em planta) & \multicolumn{2}{|l|}{$478,48 \mathrm{~m}$} \\
\hline & & Comprimento médio (em elevação) & \multicolumn{2}{|l|}{$521,39 \mathrm{~m}$} \\
\hline & \multirow{4}{*}{$\begin{array}{c}\text { POSICIONAMENTO } \\
\text { TOPOGRÁFICO } \\
\text { (por segmento) }\end{array}$} & Alta vertente & $25.331,99 \mathrm{~m}^{2}$ & $50,42 \%$ \\
\hline & & Média vertente & $7.232,24 \mathrm{~m}^{2}$ & $14,40 \%$ \\
\hline & & Baixa vertente & $17.675,70 \mathrm{~m}^{2}$ & $35,18 \%$ \\
\hline & & Total & $50.239,94 \mathrm{~m}^{2}$ & $100,00 \%$ \\
\hline \multirow{25}{*}{ MOVIMENTO DE MASSA } & \multirow{9}{*}{$\begin{array}{c}\text { TOTAL } \\
\text { (raiz e depósito) }\end{array}$} & Extensão longitudinal & $111,15 \mathrm{~m}$ & \\
\hline & & Extensão lateral & $51,03 \mathrm{~m}$ & \\
\hline & & Porção em alta vertente & $0,00 \mathrm{~m}^{2}$ & $0,00 \%$ \\
\hline & & Porção em média vertente & $3.289,37 \mathrm{~m}^{2}$ & $6,55 \%$ \\
\hline & & Porção em baixa vertente & $3.129,43 \mathrm{~m}^{2}$ & $6,23 \%$ \\
\hline & & Total (raiz e depósito) & $6.418,80 \mathrm{~m}^{2}$ & $12,78 \%$ \\
\hline & & Raiz ou Cicatriz & $1.491,67 \mathrm{~m}^{2}$ & $2,97 \%$ \\
\hline & & Depósito & $4.927,14 \mathrm{~m}^{2}$ & $9,81 \%$ \\
\hline & & Superfície de efetivo potencial de ruptura SEPR* $(F<1)$ & $2.903,66 \mathrm{~m}^{2}$ & $5,78 \%$ \\
\hline & \multirow{12}{*}{ DEPÓSITO } & Espessura média & $7,63 \mathrm{~m}$ & \\
\hline & & Volume & $36.712,77 \mathrm{~m}^{3}$ & \\
\hline & & Volume (em piscinas olímpicas) & 14,69 р.0 & \\
\hline & & Densidade aparente do solo drenado & $1,15 \mathrm{t} / \mathrm{m}^{3}$ & \\
\hline & & Densidade aparente total do solo úmido & $1,59 \mathrm{t} / \mathrm{m}^{3}$ & \\
\hline & & Densidade dos sólidos & $2,65 \mathrm{t} / \mathrm{m}^{3}$ & \\
\hline & & Massa mobilizada (sólidos) & $41.840,54 \mathrm{t}$ & \\
\hline & & Água total mobilizada (gravitacional e bolsões texturais) & $16.648,00 \mathrm{t}$ & \\
\hline & & Peso total do material mobilizado (sólidos e água) & $58.488,54 \mathrm{t}$ & \\
\hline & & Peso da água retida nos bolsões texturais & $5.823,74 \mathrm{t}$ & \\
\hline & & Extensão longitudinal & $85,32 \mathrm{~m}$ & \\
\hline & & Extensão lateral & $44,75 \mathrm{~m}$ & \\
\hline & \multirow{4}{*}{ COBERTURA VEGETAL } & Peso total mobilizado (cicatriz e depósito) & $7.754,32 \mathrm{t}$ & \\
\hline & & Carga sobre a "SEPR" & $3.145,80 \mathrm{t}$ & \\
\hline & & Densidade média na "SEPR" & $0,07 \mathrm{t} / \mathrm{m}^{3}$ & \\
\hline & & Estatura média do dossel & $15,74 \mathrm{~m}$ & \\
\hline
\end{tabular}




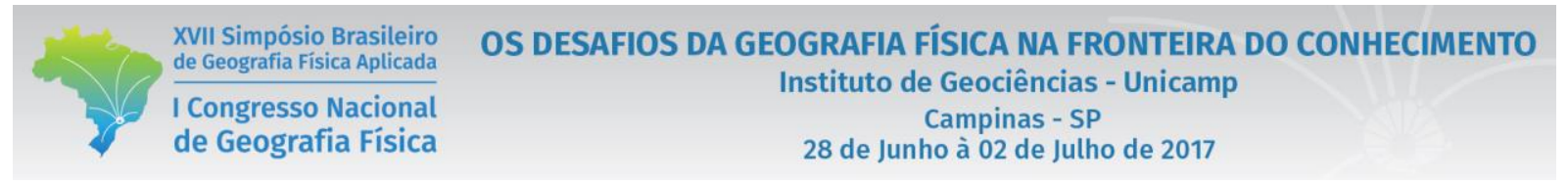

Tabela 2 - Parâmetros morfológicos, geotécnicos e hidrológicos vinculados à "superfície de efetivo potencial de ruptura" no protótipo de movimento de massa gerado pelo experimento 455.

\begin{tabular}{|c|c|c|c|}
\hline \multirow{9}{*}{$\begin{array}{c}\text { SUPERFÍCIE DE RUPTURA } \\
\text { POTENCIAL "SRP" } \\
\text { PARÂMETROS : }\end{array}$} & MORFOLÓGICOS & $\begin{array}{l}\text { Declividade média da "SRP" }\left(\alpha_{\mathrm{m}}\right) \\
\text { Declividade máxima da "SRP" }\left(\alpha_{\max }\right)\end{array}$ & $\begin{array}{l}27,52 \text { graus } \\
42,89 \text { graus } \\
\end{array}$ \\
\hline & \multirow{6}{*}{$\begin{array}{c}\text { GEOTÉCNICOS } \\
\text { (valores médios, quando } \\
\text { não especifiado) }\end{array}$} & Porção da "SRP" com débito de fricção $(\phi-\alpha<0)$ & $1.205,60 \mathrm{~m}^{2}$ \\
\hline & & Declividade média da porção da "SRP" com débito de fricção & 41,18 graus \\
\hline & & Ângulo de fricção interna médio na área com débito de fricção & 39,75 graus \\
\hline & & Débito médio de fricção na SRP $(\phi-\alpha<0)$ & $-1,43$ graus \\
\hline & & Débito máximo de fricção na SRP $(\phi-\alpha<0)$ & $-3,07$ graus \\
\hline & & Porosidade média na "SRP" & $0,53 \%$ \\
\hline & \multirow{2}{*}{$\begin{array}{l}\text { HIDROLÓGICOS } \\
\text { (valores médios) }\end{array}$} & Nível relativo de saturação na SRP $(0<m<1)$ & 0,62 \\
\hline & & Declividade do topo do fluxo de saturação na "SPR"( $\theta)$ & 27,14 graus \\
\hline \multirow{26}{*}{$\begin{array}{c}\text { SUPERFÍCIE DE EFETIVO } \\
\text { POTENCIAL DE RUPTURA } \\
\text { "SEPR" } \\
\left(\mathrm{F}_{\mathrm{s}}<1\right) \\
\text { PARÂMETROS : }\end{array}$} & \multirow{5}{*}{ MORFOLÓGICOS } & Área da "SEPR" & $2.903,66 \mathrm{~m}^{2}$ \\
\hline & & Declividade mínima na "SEPR" $\left(\alpha_{\min }\right)$ & 30,73 graus \\
\hline & & Declividade média na "SEPR" $\left(\alpha_{\mathrm{m}}\right)$ & 34,42 graus \\
\hline & & Declividade máxima na "SEPR" $\left(\alpha_{\max }\right)$ & 38,19 graus \\
\hline & & Espessura média do material sobrejacente à "SEPR" $\left(\mathrm{Z}_{\mathrm{m}}\right)$ & $10,29 \mathrm{~m}$ \\
\hline & \multirow{18}{*}{$\begin{array}{c}\text { GEOTÉCNICOS } \\
\text { (valores médios, quando } \\
\text { não especificado) }\end{array}$} & Espessura crítica do material sobrejacente à "SEPR" (Zc) & $9,82 \mathrm{~m}$ \\
\hline & & Porosidade média do meterial na "SEPR" & $0,54 \%$ \\
\hline & & Densideade aparente do solo úmido na "SEPR" & $1,60 \mathrm{t} / \mathrm{m}^{3}$ \\
\hline & & Coesão (c) & $27,63 \mathrm{KPa}$ \\
\hline & & Ângulo de fricção interna $(\phi)$ & 42,58 graus \\
\hline & & Tensão cisalhante $(\tau)$ & $670,97 \mathrm{KPa}$ \\
\hline & & Resistência ao cisalhamento (s) & $657,07 \mathrm{KPa}$ \\
\hline & & Fator de segurança médio $\left(F_{s}\right)$ & 0,98 \\
\hline & & Fator de segurança mínimo $\left(F_{s}\right)$ & 0,96 \\
\hline & & Limiar de declividade mínimo ( $\left.\alpha_{\text {Lmin }}\right)$ & 30,65 graus \\
\hline & & Limiar de declividade médio $\left(\alpha_{\mathrm{Lm}}\right)$ & 33,86 graus \\
\hline & & Limiar de declividade máximo ( $\left.\alpha_{\text {Lmax }}\right)$ & 38,13 graus \\
\hline & & Débito médio de resistência ao cisalhamento $(s-\tau)$ & $-13,90 \mathrm{KPa}$ \\
\hline & & Débito máximo de resistência ao cisalhamento $(s-\tau<0)$ & $-24,85 \mathrm{KPa}$ \\
\hline & & Débito/Crédito de fricção na "SEPR" $(\phi-\alpha)$ & 8,16 graus \\
\hline & & Porção da SEPR com débito de fricção $(\phi-\alpha<0)$ & $0,00 \mathrm{~m}^{2}$ \\
\hline & & Percentagem da SEPR com débito de fricção & $0,00 \%$ \\
\hline & & Débito máximo de fricção na "SEPR" & 0,00 graus \\
\hline & \multirow{3}{*}{$\begin{array}{l}\text { HIDROLÓGICOS } \\
\text { (valores médios) }\end{array}$} & Nível relativo de saturação na "SEPR" $(0<m<1)$ & 0,80 \\
\hline & & Declividade do topo do fluxo de saturação na "SEPR" $(\theta)$ & 34,09 graus \\
\hline & & Declividade do topo do fluxo de saturação na "SPR" $(\theta)$ & 27,14 graus \\
\hline
\end{tabular}

O fato acima descrito, está associado à atuação sinergética de dois fatores: níveis mais altos de saturação hídrica do solo associados aos setores basais e menores declividades nesses setores de encosta que, por esta mesma razão tendem a estar vinculados a superfícies de ruptura potenciais mais profundas (Anderson et all. , 1996).

Podemos admitir a possibilidade de que quadros paramétricos similares aos aqui descritos, possam se configurar em encostas reais, uma vez que a condutividade hidráulica nos cambissolos, frequentemente associados a porções de encosta muito declivosas, costuma ser elevada. Isto se deve ao fato de seus materiais serem texturalmente mais grosseiros, com baixo percentual de argila (Terzaghi; Peck, 1967). 


\section{OS DESAFIOS DA GEOGRAFIA FÍSICA NA FRONTEIRA DO CONHECIMENTO \\ Instituto de Geociências - Unicamp \\ Campinas - SP \\ 28 de Junho à 02 de Julho de 2017}

Sem dúvida, parametrizações feitas com a ajuda desses dispositivos não expressam necessariamente uma verdade empírica específica. Entretanto, sua utilidade reside justamente no fato de permitirem a realização imediata de experimentos envolvendo um grande número de variáveis, o que ainda não pode ser feito de outra forma.

\section{Símbolos e notação}

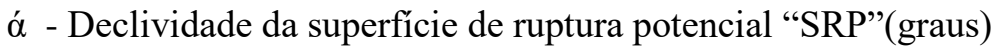

$\alpha_{c}$ - Declividade crítica da "SRP”(graus)

$\beta$ - Declividade da vertente (graus)

$\gamma_{S}$ - Peso específico do solo $\left(\mathrm{Kgf} / \mathrm{m}^{3}\right)$

$\gamma_{V}$ - Peso específico da cobertura vegetal $\left(\mathrm{Kgf} / \mathrm{m}^{3}\right)$

$\theta$ - Declividade do topo do fluxo hídrico de percolação (graus)

$\varphi^{\prime}$ - Ângulo de fricção interna efetivo (graus)

$\sigma$ ' - "Stress" efetivo $(\sigma$ ' $=\sigma-\mathrm{u}$, em KPa, KN/m² $)$

$\rho_{\mathrm{S}}$ - Massa específica do solo úmido $\left(\mathrm{Kg} / \mathrm{m}^{3}\right)$

$\rho_{\mathrm{V}}$ - Massa específica da vegetação $\left(\mathrm{Kg} / \mathrm{m}^{3}\right)$

$\tau$ - Tensão cisalhante (em $\mathrm{KPa}, \mathrm{KN} / \mathrm{m}^{2}$ )

c' - Coesão efetiva (graus)

$\mathrm{g}$ - Aceleração da gravidade $(9,806 \mathrm{~m} / \mathrm{s})$

$\mathrm{h}_{\mathrm{V}}$ - Altura da cobertura vegetal (metros)

$\mathrm{m}$ - Nível relativo de saturação do solo $(0 \beta \mathrm{m} \beta$ 1)

$\mathrm{m}_{\mathrm{C}}$ - Nível crítico de saturação do solo $(0 \beta \mathrm{m} \beta$ 1)

$\mathrm{n}$ - Fator de retensão de água no solo $(0 \beta \mathrm{n} \beta 1$, sendo $\mathrm{n}<<1)$

$\mathrm{s}$ - Resistência ao cisalhamento $\left(\mathrm{KPa}, \mathrm{KN} / \mathrm{m}^{2}\right)$

u - Tensão neutra da água $\left(\mathrm{KPa}, \mathrm{KN} / \mathrm{m}^{2}\right)$

w - Projeção da inclinação do topo do fluxo de percolação em relação à declividade da SRP, descrita pela

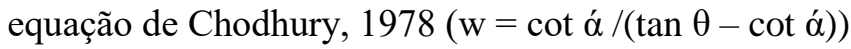

$\mathrm{Z}$ - Profundidade do material até a "SRP" (metros)

$\mathrm{Z}_{\mathrm{C}}$ - Profundidade crítica do material até a "SRP" (metros)

\section{Bibliografia}

ANDERSON, M. G. ; RICHARDS, K. S. (1987) Slope Stability: Geotechnical, Engineering and Geomorphology, John Wiley \& Sons, Chichester, 648p

ANDERSON, M. G.; COLLISON, A. J. C.; HARTSHORNE, J.; LlOYD, D. M. (1996) Developments in Slope Hydrology-Stability Modelling for Tropical Slopes. In Advances in Hillslope Processes, Malcolm G. Anderson \& Susan M. Brooks eds., vol.2, pp.799-822.

CARSON, M. A. (1975) Thresholds and característic angles of straight slopes. in Mass Wasting, 4th Ghelph symposium on Geomorphology, Ontario. 
COLANGELO, A.C. Geomorfossíntese e Geomorfocinemática aplicadas à evolução e estabilidade de vertentes. 2007, 228 f. Tese (Livre Docência) - Faculdade de Filosofia Letras e Ciências Humanas, São Paulo.

COLANGELO, A. C. - 2008 - Relief unity emulator applied to slope stability analysis, Geophysical Research Abstracts, Vol. 10, European Geosciences Union - General Assembly, EGU2008-A-00850, NH4.13-1WE10-005, Oral presentation, Session - Landslides risk assessment methods strategies, Apr. $14^{\text {th }}$ to $19^{\text {th }}$,Vienna, Austria.

COLANGELO, A. C. - 2009 - Physical and morphological analysis of synthetic mass movements generated by slope stability simulator, 8th IAG International Conference on Geomorphology, Oral presentation, Sessão Hillslopes and Mass Movements - July 6th to 11 th, Melbourne, Australia

COLANGELO, A. C. - 2012 - Os Parâmetros de Resistência ao Cisalhamento e a Estabilidade das Encostas no Planalto de Paraibuna e Serra de Caraguatatuba, Revista do Departamento de Geografia - USP, (ISSN 2236-2878 DOI: 10.7154) Volume Especial 30 Anos (2012), p. 112-129.

GEUZE, E.C.W.A. (1963) The uniqueness of the Mohr-Coulomb concept in shear failure. In ASTM Special Technical Publication n ${ }^{\mathrm{o}}$ 361, Philadelphia, $\mathrm{Pa}$ - USA, pp52-64.

HVORSlEV, J. (1936) Conditions for Failure of Remolded Cohesive Soils, Proc. First Int. Conf. of Soil Mech. And Found. Eng., Cambridge.

TERZAGUI, K.; PECK, R. B. (1967) Soil mechanics in engineering practice. Wiley, New York, 729 p. 